International Journal of Applied Mathematics

Volume 34 No. $2 \quad 2021,339-351$

ISSN: $1311-1728$ (printed version); ISSN: 1314-8060 (on-line version)

doi: http://dx.doi.org/10.12732/ijam.v34i2.10

\title{
ELLIPTIC EQUATIONS IN DOMAINS WITH CUTS: CERTAIN EXAMPLES
}

\author{
Vladimir Vasilyev $^{1 \S}$, Shaban Kutaiba ${ }^{2}$ \\ ${ }^{1,2}$ Chair of Applied Mathematics \\ and Computer Modeling \\ Belgorod State National Research University \\ Pobedy street 85, Belgorod - 308015, RUSSIA
}

\begin{abstract}
We consider some special examples of boundary value problems for a model elliptic pseudo-differential equation in a special cone in 3-dimensional space. Using a concept of wave factorization for an elliptic symbol and the formula for a general solution for the equation we study limit behavior of the solution when some parameters of the cone tend to their limit values.
\end{abstract}

AMS Subject Classification: 35S15, 58J05

Key Words: model elliptic pseudo-differential equation; a cone; solvability condition

\section{Introduction}

The theory of boundary value problems for elliptic pseudo-differential equations for domains with a smooth boundary are completely presented in the book [3]. Based on this book, a theory of pseudo-differential operators [5] and multidimensional complex analysis [1], [2], the first author suggested analogous approach for manifolds with conical singularities at a boundary [8]. There are different approaches for studying pseudo-differential operators and equations on non-smooth manifolds and a lot of applications [9], [10], [11], [12], [13], but our approach is based on a concept of wave factorization for an elliptic symbol $[8]$.

Received: December 15, 2020

(C) 2021 Academic Publications

${ }^{\S}$ Correspondence author 
Here we will consider some consequences from the results in [14], [15], [16], related to special boundary value problem for a model elliptic pseudodifferential equation in a cone. These studies were inspired by more earlier papers of the first author and lead to different boundary singularities of the domain (manifold) and enlarge a class of manifolds under consideration. First, in this section we introduce some necessary concepts and definitions which are needed for further studying. We widely use the theory of one-dimensional singular integral operators [4], [6], [7], and our constructions are closely related to such operators.

\subsection{Canonical Domains and Model Equations}

By canonical domain $D \subset \mathbf{R}^{m}$ we mean one of the following domains: $\mathbf{R}^{m}$, a half-space $\mathbf{R}_{+}^{m}=\left\{x \in \mathbf{R}^{m}: x=\left(x^{\prime}, x_{m}\right), x_{m}>0\right\}$ or a certain cone in $\mathbf{R}^{m}$.of a type $\mathbf{R}^{k} \times C^{m-k}$, where $C^{m-k}$ is a convex cone in $\mathbf{R}^{m-k}$ non-including any whole straight line, $0 \leq k \leq m$. By definition, $\mathbf{R}^{0} \times C^{m} \equiv C^{m}, \mathbf{R}^{m} \times C^{0} \equiv \mathbf{R}^{\mathbf{m}}$.

The first two cases were studied earlier [3], [6] and here we concentrated on the left case with a cone. More exactly, in this paper we consider special cones.

A model pseudo-differential operator in a canonical domain $D$ is defined in the following way

$$
(A u)(x)=\int_{D} \int_{\mathbf{R}^{m}} A(\xi) u(y) e^{i(y-x) \cdot \xi} d \xi d y, \quad x \in D,
$$

where the function $A(\xi)$ is called a symbol of the pseudo-differential operator $A$. We use the term "model" because the considered symbol does not depend on a spatial variable $x$. For more general situations they consider symbols of the type $A(x, \xi)$. Our restriction is explained by so called local principle; it asserts roughly speaking that we need to obtain invertibility conditions for a model operator for describing Fredholm properties for an operator with a general symbol $A(x, \xi)$.

We will consider the class of symbols satisfying the condition

$$
c_{1} \leq\left|A(\xi)(1+|\xi|)^{-\alpha}\right| \leq c_{2}, \quad \xi \in \mathbf{R}^{m} .
$$

The number $\alpha \in \mathbf{R}$ we call an order of a pseudo-differential operator $A$.

It is well known that such an operator is a linear bounded operator acting from the space $H^{s}\left(\mathbf{R}^{m}\right)$ into the space $H^{s-\alpha}\left(\mathbf{R}^{m}\right)$ and invertible [3]. Our mail goal is describing invertibility conditions for such operator in different canonical domains $D$. 
Let $C$ be a convex cone in the space $\mathbf{R}^{\mathbf{m}}$, and this cone does not include any whole straight line, it is important because we use the theory of analytic functions of several complex variables [1], [2]. Moreover we suppose that a surface of this cone is given by the equation $x_{m}=\varphi\left(x^{\prime}\right), x^{\prime}=\left(x_{1}, \cdots, x_{m-1}\right)$, where $\varphi: \mathbf{R}^{\mathbf{m}-\mathbf{1}} \rightarrow \mathbf{R}$ is a smooth function in $\mathbf{R}^{\mathbf{m}-\mathbf{1}} \backslash\{\mathbf{0}\}$, and $\varphi(0)=0$.

Thus, we study a solvability of the model pseudo-differential equation [8], [14], [15]

$$
\left(A u_{+}\right)(x)=f(x), x \in C,
$$

in the space $H^{s}(C)$, where $A$ is a pseudo-differential operator with the symbol $A(\xi)$ satisfying the condition (1). The sign "+" for the unknown function $u_{+}$ shows that this function is defined in $C+$ only. We will remind some general concepts.

By definition the space $H^{s}\left(C_{+}^{a}\right)$ consists of functions from the space $H^{s}\left(\mathbf{R}^{m}\right)$ with supports in $\overline{C_{+}^{a}}$. A norm in the space $H^{s}\left(C_{+}^{a}\right)$ is induced by a norm of the space $H^{s}\left(\mathbf{R}^{m}\right)$.

Let us denote by $S\left(\mathbf{R}^{\mathbf{m}}\right)$ the Schwartz class of infinitely differentiable rapidly decreasing at infinity functions, $S^{\prime}\left(\mathbf{R}^{\mathbf{m}}\right)$ is a space of distributions over $S\left(\mathbf{R}^{\mathbf{m}}\right)$, and $\left.S_{+}^{\prime a}\right)$ is a space of distributions from $S^{\prime}\left(\mathbf{R}^{\mathbf{m}}\right)$ with supports in $\overline{C_{+}^{a}} \cdot f$ in the right hand side in the equation (1) is taken from the space $H_{0}^{s-\alpha}\left(C_{+}^{a}\right)$ consisting of distributions from $S_{+}^{\prime a}$ ) which admit a continuation into a whole space $H^{s-\alpha}\left(\mathbf{R}^{m}\right)$. A norm in the space $H_{0}^{s-\alpha}\left(C_{+}^{a}\right)$ is defined by the formula

$$
\|f\|_{s-\alpha}^{+}=\inf \|l f\|_{s-\alpha}
$$

where infimum is taken over all continuations $l f$.

\subsection{Wave Factorization and Solution Constructions}

Definition 1. A radial tube domain over the cone $C$ is called a domain in 3-dimensional complex space $\mathbf{C}^{m}$ of the following type

$$
T(C) \equiv\left\{z \in \mathbf{C}^{m}: z=x+i y, x \in \mathbf{R}^{m}, y \in C\right\} .
$$

A conjugate cone $\stackrel{*}{C}$ is called such a cone in which for all points the condition

$$
x \cdot y>0, \quad \forall y \in C,
$$

holds; $x \cdot y$ means inner product for $x$ and $y$. 
Definition 2. The wave factorization of an elliptic symbol $A(\xi)$ with respect to the cone $C$ is called its representation in the form

$$
A(\xi)=A_{\neq}(\xi) A_{=}(\xi)
$$

where factors $A_{\neq}(\xi), A_{=}(\xi)$ must satisfy the following conditions:

1) $A_{\neq}(\xi), A_{=}(\xi)$ are defined for all $\xi \in \mathbf{R}^{m}$ may be except the points $\xi \in \partial \stackrel{*}{C}$;

2) $A_{\neq}(\xi), A_{=}(\xi)$ admit an analytic continuation into radial tube domains $T(\stackrel{*}{C}), T(-\stackrel{*}{C})$ respectively with estimates

$$
\begin{gathered}
\left|A_{\neq}^{ \pm 1}(\xi+i \tau)\right| \leq c_{1}(1+|\xi|+|\tau|)^{ \pm æ}, \\
\left|A_{=}^{ \pm 1}(\xi-i \tau)\right| \leq c_{2}(1+|\xi|+|\tau|)^{ \pm(\alpha-æ)}, \forall \tau \in \stackrel{*}{C} .
\end{gathered}
$$

The number $æ \in \mathbf{R}$ is called an index of the wave factorization.

Remark 3. Let us note that below we will use some distinct definition for the wave factorization assuming that $A_{\neq}(\xi)$ admits an analytical continuation into $T(-\stackrel{*}{C})$, and $A_{=}(\xi)$ admits an analytical continuation into $T(\stackrel{*}{C})$ because we will study a problem outside a cone.

Everywhere below we consider the case $æ-s=1+\delta,|\delta|<1 / 2$ only.

\subsection{Elliptic Operators and Equations}

To describe our advances for low dimensional cones we consider the following equation

$$
(A u)(x)=0 \quad x \in \mathbf{R}^{3} \backslash C_{+}^{a b}
$$

in the Sobolev-Slobodetskii space $H^{s}\left(C_{+}^{a b}\right)$, where

$$
C_{+}^{a b}=\left\{x \in \mathbf{R}^{3}: x=\left(x_{1}, x_{2}, x_{3}\right) \cdot x_{3}<a\left|x_{1}\right|+b\left|x_{2}\right|, a, b>0\right\} .
$$

To present a general solution of the equation (2) for the case $æ-s=1+\delta$, $|\delta|<1 / 2$, we use some results from [15], [16]. Let us introduce the following one-dimensional singular integral operators [4], [6]

$$
\left(S_{1} u\right)\left(\xi_{1}, \xi_{2}, \xi_{3}\right)=v \cdot p \frac{i}{2 \pi} \int_{-\infty}^{+\infty} \frac{u\left(\tau, \xi_{2}, \xi_{3}\right) d \tau}{\xi_{1}-\tau}
$$




$$
\left(S_{2} u\right)\left(\xi_{1}, \xi_{2}, \xi_{3}\right)=v \cdot p \frac{i}{2 \pi} \int_{-\infty}^{+\infty} \frac{u\left(\xi_{1}, \eta, \xi_{3}\right) d \eta}{\xi_{2}-\eta}
$$

and write

$$
\begin{gathered}
A_{\neq}(\xi) \tilde{u}(\xi)=\tilde{C}_{1}\left(\xi_{1}-a \xi_{3}, \xi_{2}-b \xi_{3}\right)+\tilde{C}_{2}\left(\xi_{1}-a \xi_{3}, \xi_{2}+b \xi_{3}\right) \\
+\tilde{C}_{3}\left(\xi_{1}+a \xi_{3}, \xi_{2}-b \xi_{3}\right)+\tilde{C}_{4}\left(\xi_{1}+a \xi_{3}, \xi_{2}+b \xi_{3}\right),
\end{gathered}
$$

where

$$
\begin{aligned}
& \tilde{C}_{1}\left(\xi_{1}-a \xi_{3}, \xi_{2}-b \xi_{3}\right)= \frac{1}{4} \tilde{c}_{0}\left(\xi_{1}-a \xi_{3}, \xi_{2}-b \xi_{3}\right)-\frac{1}{2}\left(S_{1} \tilde{c}_{0}\right)\left(\xi_{1}-a \xi_{3}, \xi_{2}-b \xi_{3}\right) \\
&-\frac{1}{2}\left(S_{2} \tilde{c}_{0}\right)\left(\xi_{1}-a \xi_{3}, \xi_{2}-b \xi_{3}\right)+\left(S_{1} S_{2} \tilde{c}_{0}\right)\left(\xi_{1}-a \xi_{3}, \xi_{2}-b \xi_{3}\right), \\
& \tilde{C}_{2}\left(\xi_{1}-a \xi_{3}, \xi_{2}+b \xi_{3}\right)=\frac{1}{4} \tilde{c}_{0}\left(\xi_{1}-a \xi_{3}, \xi_{2}+b \xi_{3}\right)-\frac{1}{2}\left(S_{1} \tilde{c}_{0}\right)\left(\xi_{1}-a \xi_{3}, \xi_{2}+b \xi_{3}\right) \\
&+\frac{1}{2}\left(S_{2} \tilde{c}_{0}\right)\left(\xi_{1}-a \xi_{3}, \xi_{2}+b \xi_{3}\right)-\left(S_{1} S_{2} \tilde{c}_{0}\right)\left(\xi_{1}-a \xi_{3}, \xi_{2}+b \xi_{3}\right), \\
& \tilde{C}_{3}\left(\xi_{1}+a \xi_{3}, \xi_{2}-b \xi_{3}\right)=\frac{1}{4} \tilde{c}_{0}\left(\xi_{1}+a \xi_{3}, \xi_{2}-b \xi_{3}\right)+\frac{1}{2}\left(S_{1} \tilde{c}_{0}\right)\left(\xi_{1}+a \xi_{3}, \xi_{2}-b \xi_{3}\right) \\
&-\frac{1}{2}\left(S_{2} \tilde{c}_{0}\right)\left(\xi_{1}+a \xi_{3}, \xi_{2}-b \xi_{3}\right)-\left(S_{1} S_{2} \tilde{c}_{0}\right)\left(\xi_{1}+a \xi_{3}, \xi_{2}-b \xi_{3}\right), \\
& \tilde{C}_{4}\left(\xi_{1}+a \xi_{3}, \xi_{2}+b \xi_{3}\right)=\frac{1}{4} \tilde{c}_{0}\left(\xi_{1}+a \xi_{3}, \xi_{2}+b \xi_{3}\right)+\frac{1}{2}\left(S_{1} \tilde{c}_{0}\right)\left(\xi_{1}+a \xi_{3}, \xi_{2}+b \xi_{3}\right) \\
&+\frac{1}{2}\left(S_{2} \tilde{c}_{0}\right)\left(\xi_{1}+a \xi_{3}, \xi_{2}+b \xi_{3}\right)+\left(S_{1} S_{2} \tilde{c}_{0}\right)\left(\xi_{1}+a \xi_{3}, \xi_{2}+b \xi_{3}\right),
\end{aligned}
$$

where $c_{0}\left(x_{1}, x_{2}\right)$ is an arbitrary function from the space $H^{s-æ+1 / 2}\left(\mathbf{R}^{2}\right)$. In other words, a kernel of the operator $A$ is a one-dimensional subspace.

To determine uniquely the arbitrary function $c_{0}\left(\xi_{1}, \xi_{2}\right)$ we require certain additional condition, for example, we assume that the restriction $\tilde{u}\left(\xi_{1}, \xi_{2}, 0\right)$ is given, i.e. the following integral

$$
\int_{-\infty}^{+\infty} u\left(x_{1}, x_{2}, x_{3}\right) d x_{3} \equiv g\left(x_{1}, x_{2}\right),
$$

it gives the equality

$$
\tilde{u}\left(\xi_{1}, \xi_{2}, 0\right)=\tilde{g}\left(\xi_{1}, \xi_{2}\right) .
$$


Taking into account $\xi_{3}=0$ in the formula (3) we find

$$
\begin{gathered}
\sum_{k=1}^{4} \tilde{C}_{k}\left(\xi_{1}, \xi_{2}\right) \\
=\frac{1}{4} \tilde{c}_{0}\left(\xi_{1}, \xi_{2}\right)-\frac{1}{2}\left(S_{1} \tilde{c}_{0}\right)\left(\xi_{1}, \xi_{2}\right)-\frac{1}{2}\left(S_{2} \tilde{c}_{0}\right)\left(\xi_{1}, \xi_{2}\right)+\left(S_{1} S_{2} \tilde{c}_{0}\right)\left(\xi_{1}, \xi_{2}\right) \\
+\frac{1}{4} \tilde{c}_{0}\left(\xi_{1}, \xi_{2}\right)-\frac{1}{2}\left(S_{1} \tilde{c}_{0}\right)\left(\xi_{1}, \xi_{2}\right)+\frac{1}{2}\left(S_{2} \tilde{c}_{0}\right)\left(\xi_{1}, \xi_{2}\right)-\left(S_{1} S_{2} \tilde{c}_{0}\right)\left(\xi_{1}, \xi_{2}\right) \\
+\frac{1}{4} \tilde{c}_{0}\left(\xi_{1}, \xi_{2}\right)+\frac{1}{2}\left(S_{1} \tilde{c}_{0}\right)\left(\xi_{1}, \xi_{2}\right)-\frac{1}{2}\left(S_{2} \tilde{c}_{0}\right)\left(\xi_{1}, \xi_{2}\right)-\left(S_{1} S_{2} \tilde{c}_{0}\right)\left(\xi_{1}, \xi_{2}\right) \\
+\frac{1}{4} \tilde{c}_{0}\left(\xi_{1}, \xi_{2}\right)+\frac{1}{2}\left(S_{1} \tilde{c}_{0}\right)\left(\xi_{1}, \xi_{2}\right)+\frac{1}{2}\left(S_{2} \tilde{c}_{0}\right)\left(\xi_{1}, \xi_{2}\right)+\left(S_{1} S_{2} \tilde{c}_{0}\right)\left(\xi_{1}, \xi_{2}\right) \\
=\tilde{c}_{0}\left(\xi_{1}, \xi_{2}\right) .
\end{gathered}
$$

In view of the condition (5), we obtain

$$
\tilde{c}_{0}\left(\xi^{\prime}\right)=\tilde{A}_{\neq}\left(\xi^{\prime}, 0\right) \tilde{g}\left(\xi^{\prime}\right) .
$$

Theorem 4. Let $æ-s=1+\delta,|\delta|<1 / 2, g \in H^{s+1 / 2}\left(\mathbf{R}^{2}\right)$. Then the unique solution of the problem (2),(4) is given by the formula (3), and $c_{0}\left(x_{1}, x_{2}\right)$ is determined by the formula (6).

The proof of Theorem 4 is given in details in [15], [16].

Further, our main goal is describing behavior of the unique solution of the problem $(2),(4)$ when the parameters $a, b$ tend to their endpoint values, 0 and $\infty$. Of course, we will assume that the needed wave factorization exists for enough small (large) values. Let us note that the cases for some small values $a, b$ were studied in [16].

In the next section we will consider some domains in Euclidean space $\mathbf{R}^{3}$ that we can obtain under endpoint values of the parameters. Such domains we call domains with cuts. In the plane case one can obtain only one type of such a domain, but in 3-dimensional space there are a few types.

We will show below certain simple examples. Based on the formula (3) we find certain condition for the boundary function $g$. 


\section{Limit Boundary Value Problems}

\subsection{A half-plane in a space}

In the previous section we have two parameters of the cone $a$ and $b$. Degenerated case corresponds to that case when one of the parameters (or both) tends to 0 or $+\infty$. Let us note that case $a, b \rightarrow 0$ was studied in [3], the cases $a \rightarrow 0, b=$ const and $a=$ const, $b \rightarrow 0$ were studied in [16]. Here we consider limit case $a \rightarrow+\infty, b=$ const, the case $a=$ const, $b \rightarrow+\infty$ looks almost the same. Let us remind that this case corresponds to a half-plane cut in $\mathbf{R}^{3}$.

Starting point for this consideration will be the equality (3). We use the change of variables $\xi_{1}-a \xi_{3}=t_{1}, \xi_{1}+a \xi_{3}=t_{3}$ from which we have $\xi_{1}=$ $\frac{t_{3}+t_{1}}{2}, \xi_{3}=\frac{t_{3}-t_{1}}{2 a}$. So, we have new variables $t_{1}, \xi_{2}, t_{3}$. If we use the condition (5) we can find the unknown function $\tilde{c}_{0}$ by the formula (6). Now let us write the formula (3) for the new variables $t_{1}, \xi_{2}, t_{3}$. Then we obtain

$$
\begin{aligned}
& A_{\neq}\left(\frac{t_{3}+t_{1}}{2}, \xi_{2}, \frac{t_{3}-t_{1}}{2 a}\right) \tilde{u}\left(\frac{t_{3}+t_{1}}{2}, \xi_{2}, \frac{t_{3}-t_{1}}{2 a}\right) \\
& =\tilde{C}_{1}\left(t_{1}, \xi_{2}-b \frac{t_{3}-t_{1}}{2 a}\right)+\tilde{C}_{2}\left(t_{1}, \xi_{2}+b \frac{t_{3}-t_{1}}{2 a}\right) \\
& +\tilde{C}_{3}\left(t_{3}, \xi_{2}-b \frac{t_{3}-t_{1}}{2 a}\right)+\tilde{C}_{4}\left(t_{3}, \xi_{2}+b \frac{t_{3}-t_{1}}{2 a}\right) .
\end{aligned}
$$

Tending $a$ to $+\infty$ we obtain the following relation

$$
\begin{gathered}
A_{\neq}\left(\frac{t_{3}+t_{1}}{2}, \xi_{2}, 0\right) \tilde{u}\left(\frac{t_{3}+t_{1}}{2}, \xi_{2}, 0\right) \\
=\tilde{C}_{1}\left(t_{1}, \xi_{2}\right)+\tilde{+} C_{2}\left(t_{1}, \xi_{2}\right)+\tilde{C}_{3}\left(t_{3}, \xi_{2}\right)+\tilde{C}_{4}\left(t_{3}, \xi_{2}\right) .
\end{gathered}
$$

After accurate calculations we find

$$
\begin{gathered}
\tilde{C}_{1}\left(t_{1}, \xi_{2}\right)+\tilde{C}_{2}\left(t_{1}, \xi_{2}\right)+\tilde{C}_{3}\left(t_{3}, \xi_{2}\right)+\tilde{C}_{4}\left(t_{3}, \xi_{2}\right) \\
=\frac{\tilde{c}_{0}\left(t_{1}, \xi_{2}\right)+\tilde{c}_{0}\left(t_{3}, \xi_{2}\right)}{2}-\left(S_{1} \tilde{c}_{0}\right)\left(t_{1}, \xi_{2}\right)+\left(S_{1} \tilde{c}_{0}\right)\left(t_{3}, \xi_{2}\right) .
\end{gathered}
$$

Taking into account the condition (6), the formula (7) and new notation

$$
\tilde{A}_{\neq}\left(\xi_{1}, \xi_{2}, 0\right) \tilde{g}\left(\xi_{1}, \xi_{2}\right) \equiv h\left(\xi_{1}, \xi_{2}\right),
$$


we obtain the following equation with parameter $\xi_{2}$

$$
\begin{gathered}
h\left(\frac{t_{3}+t_{1}}{2}, \xi_{2}\right)=\frac{h\left(t_{1}, \xi_{2}\right)+h\left(t_{3}, \xi_{2}\right)}{2} \\
-\left(S_{1} h\right)\left(t_{1}, \xi_{2}\right)+\left(S_{1} h\right)\left(t_{3}, \xi_{2}\right)
\end{gathered}
$$

Thus, we have the following property.

Theorem 5. If the symbol $A(\xi)$ admits the wave factorization with respect to $C_{+}^{a b}$ with the index $æ$ such that $æ-s=1+\delta,|\delta|<1 / 2$ for enough large $a$ then the unique solution of the boundary value problem (2),(4) has a limit under $a \rightarrow+\infty$ if and only if the boundary function $g \in H^{s+1 / 2}\left(\mathbf{R}^{2}\right)$ satisfies the equation (8).

Remark 6. The same results are valid if $b=0, a \rightarrow \infty$ and if $a, b \rightarrow \infty$ but $\lim \frac{b}{a}=0$.

Let us introduce four operators defined by formulas

$$
\begin{aligned}
& \left(W_{1} h\right)\left(\xi_{1}, \xi_{2}\right)=\frac{1}{4} h\left(\xi_{1}, \xi_{2}\right)-\frac{1}{2}\left(S_{1} h\right)\left(\xi_{1}, \xi_{2}\right) \\
& -\frac{1}{2}\left(S_{2} h\right)\left(\xi_{1}, \xi_{2}\right)+\left(S_{1} S_{2} h\right)\left(\xi_{1}, \xi_{2}\right), \\
& \left(W_{2} h\right)\left(\xi_{1}, \xi_{2}\right)=\frac{1}{4} h\left(\xi_{1}, \xi_{2}\right)-\frac{1}{2}\left(S_{1} h\right)\left(\xi_{1}, \xi_{2}\right) \\
& +\frac{1}{2}\left(S_{2} h\right)\left(\xi_{1}, \xi_{2}\right)-\left(S_{1} S_{2} h\right)\left(\xi_{1}, \xi_{2}\right), \\
& \left(W_{3} h\right)\left(\xi_{1}, \xi_{2}\right)=\frac{1}{4} h\left(\xi_{1}, \xi_{2}\right)+\frac{1}{2}\left(S_{1} h\right)\left(\xi_{1}, \xi_{2}\right) \\
& -\frac{1}{2}\left(S_{2} h\right)\left(\xi_{1}, \xi_{2}\right)-\left(S_{1} S_{2} h\right)\left(\xi_{1}, \xi_{2}\right), \\
& \left(W_{4} h\right)\left(\xi_{1}, \xi_{2}\right)=\frac{1}{4} h\left(\xi_{1}, \xi_{2}\right)+\frac{1}{2}\left(S_{1} h\right)\left(\xi_{1}, \xi_{2}\right) \\
& +\frac{1}{2}\left(S_{2} h\right)\left(\xi_{1}, \xi_{2}\right)+\left(S_{1} S_{2} h\right)\left(\xi_{1}, \xi_{2}\right) .
\end{aligned}
$$

Corollary 7. If $a=b \rightarrow \infty$, then we obtain the following relation for the function $\tilde{g}\left(\xi_{1}, \xi_{2}\right)$

$$
\begin{gathered}
h\left(\frac{t_{3}+t_{1}}{2}, \xi_{2}\right)=\left(W_{1} h\right)\left(t_{1}, \xi_{2}-\frac{t_{3}-t_{1}}{2}\right)+\left(W_{2} h\right)\left(t_{1}, \xi_{2}+\frac{t_{3}-t_{1}}{2}\right) \\
+\left(W_{3} h\right)\left(t_{3}, \xi_{2}-\frac{t_{3}-t_{1}}{2}\right)+\left(W_{4} h\right)\left(t_{3}, \xi_{2}+\frac{t_{3}-t_{1}}{2}\right) .
\end{gathered}
$$


Proof. We put $a=b$ in the formula (7) and consider $a \rightarrow \infty$. Then we have from $(7)$

$$
\begin{gathered}
\quad A_{\neq}\left(\frac{t_{3}+t_{1}}{2}, \xi_{2}, 0\right) \tilde{u}\left(\frac{t_{3}+t_{1}}{2}, \xi_{2}, 0\right) \\
=\tilde{C}_{1}\left(t_{1}, \xi_{2}-\frac{t_{3}-t_{1}}{2}\right)+\tilde{C}_{2}\left(t_{1}, \xi_{2}+\frac{t_{3}-t_{1}}{2}\right) \\
+\tilde{C}_{3}\left(t_{3}, \xi_{2}-\frac{t_{3}-t_{1}}{2}\right)+\tilde{C}_{4}\left(t_{3}, \xi_{2}+\frac{t_{3}-t_{1}}{2}\right) .
\end{gathered}
$$

Then we have the following equation for the function $g\left(\xi_{1}, \xi_{2}\right)$, and we remind here that $\left.h\left(\xi_{1}, \xi_{2}\right)=A_{\neq}\left(\xi_{1}, \xi_{2}, 0\right) g\left(\xi_{1}, \xi_{2}\right)\right)$ :

$$
\begin{gathered}
h\left(\frac{t_{3}+t_{1}}{2}, \xi_{2}\right)=\left(W_{1} h\right)\left(t_{1}, \xi_{2}-\frac{t_{3}-t_{1}}{2}\right)+\left(W_{2} h\right)\left(t_{1}, \xi_{2}+\frac{t_{3}-t_{1}}{2}\right) \\
+\left(W_{3} h\right)\left(t_{3}, \xi_{2}-\frac{t_{3}-t_{1}}{2}\right)+\left(W_{4} h\right)\left(t_{3}, \xi_{2}+\frac{t_{3}-t_{1}}{2}\right) .
\end{gathered}
$$

We observe the same situation when $a=$ const, $b \rightarrow \infty$. Analogously we change variables $t_{2}=\xi_{2}-b \xi_{3}, t_{3}=\xi_{2}+b \xi_{3}$. Therefore, $\xi_{2}=\frac{t_{3}+t_{2}}{2}, \xi_{3}=\frac{t_{3}-t_{2}}{2 b}$. Then we have

$$
\begin{aligned}
& A_{\neq}\left(\xi_{1}, \frac{t_{3}+t_{2}}{2}, \frac{t_{3}-t_{2}}{2 b}\right) \tilde{u}\left(\xi_{1}, \frac{t_{3}+t_{2}}{2}, \frac{t_{3}-t_{2}}{2 b}\right) \\
= & \tilde{C}_{1}\left(\xi_{1}-a \frac{t_{3}-t_{2}}{2 b}, t_{2}\right)+\tilde{C}_{2}\left(\xi_{1}-a \frac{t_{3}-t_{2}}{2 b}, t_{3}\right) \\
+ & \tilde{C}_{3}\left(\xi_{1}+a \frac{t_{3}-t_{2}}{2 b}, t_{2}\right)+\tilde{C}_{4}\left(\xi_{1}+a \frac{t_{3}-t_{2}}{2 b}, t_{3}\right) .
\end{aligned}
$$

If $b$ tends to $+\infty$, we get

$$
\begin{gathered}
A_{\neq}\left(\xi_{1}, \frac{t_{3}+t_{2}}{2}, 0\right) \tilde{u}\left(\xi_{1}, \frac{t_{3}+t_{2}}{2}, 0\right) \\
=\tilde{C}_{1}\left(\xi_{1}, t_{2}\right)+\tilde{+} C_{2}\left(\xi_{1}, t_{2}\right)+\tilde{C}_{3}\left(\xi_{1}, t_{2}\right)+\tilde{C}_{4}\left(\xi_{1}, t_{2}\right) .
\end{gathered}
$$

Simple calculations lead to the following relation:

$$
\tilde{C}_{1}\left(\xi_{1}, t_{2}\right)+\tilde{+} C_{2}\left(\xi_{1}, t_{2}\right)+\tilde{C}_{3}\left(\xi_{1}, t_{2}\right)+\tilde{C}_{4}\left(\xi_{1}, t_{2}\right)
$$




$$
=\frac{\tilde{c}_{0}\left(\xi_{1}, t_{2}\right)+\tilde{c}_{0}\left(\xi_{1}, t_{3}\right)}{2}-\left(S_{2} \tilde{c}_{0}\right)\left(\xi_{1}, t_{2}\right)+\left(S_{2} \tilde{c}_{0}\right)\left(\xi_{1}, t_{3}\right) .
$$

In view of the condition (6), the formula (9) and the notation

$$
\tilde{A}_{\neq}\left(\xi_{1}, \xi_{2}, 0\right) \tilde{g}\left(\xi_{1}, \xi_{2}\right) \equiv h\left(\xi_{1}, \xi_{2}\right),
$$

we obtain the following equation with parameter $\xi_{1}$

$$
\begin{gathered}
h\left(\xi_{1}, \frac{t_{3}+t_{2}}{2}\right)=\frac{h\left(\xi_{1}, t_{2}\right)+h\left(\xi_{1}, t_{3}\right)}{2} \\
-\left(S_{2} h\right)\left(\xi_{1}, t_{2}\right)+\left(S_{2} h\right)\left(\xi_{1}, t_{3}\right)
\end{gathered}
$$

Hence, we obtain the following result.

Theorem 8. If the symbol $A(\xi)$ admits the wave factorization with respect to $C_{+}^{a b}$ with the index æ such that $æ-s=1+\delta,|\delta|<1 / 2$ for enough large $b$, then the unique solution of the boundary value problem (2),(4) has a limit under $b \rightarrow+\infty$ if and only if the boundary function $g \in H^{s+1 / 2}\left(\mathbf{R}^{2}\right)$ satisfies the equation (12).

Remark 9. The same results are valid if $a=0, b \rightarrow \infty$ and if $a, b \rightarrow \infty$ but $\lim \frac{a}{b}=0$.

Corollary 10. If $a=b \rightarrow \infty$, then we obtain the following relation for the function $\tilde{g}\left(\xi_{1}, \xi_{2}\right)$

$$
\begin{gathered}
h\left(\xi_{1}, \frac{t_{3}+t_{2}}{2}\right)=\left(W_{1} h\right)\left(\xi_{1}-\frac{t_{3}-t_{2}}{2}, t_{2}\right)+\left(W_{2} h\right)\left(\xi_{1}-\frac{t_{3}-t_{2}}{2}, t_{3}\right) \\
+\left(W_{3} h\right)\left(\xi_{1}+\frac{t_{3}-t_{2}}{2}, t_{2}\right)+\left(W_{4} h\right)\left(\xi_{1}+\frac{t_{3}-t_{2}}{2}, t_{3}\right) .
\end{gathered}
$$

Proof. If $b \rightarrow \infty$, then we have from (11)

$$
\begin{aligned}
& A_{\neq}\left(\xi_{1}, \frac{t_{3}+t_{2}}{2}, 0\right) \tilde{u}\left(\xi_{1}, \frac{t_{3}+t_{2}}{2}, 0\right) \\
= & \tilde{C}_{1}\left(\xi_{1}-\frac{t_{3}-t_{2}}{2}, t_{2}\right)+\tilde{C}_{2}\left(\xi_{1}-\frac{t_{3}-t_{2}}{2}, t_{3}\right) \\
+ & \tilde{C}_{3}\left(\xi_{1}+\frac{t_{3}-t_{2}}{2}, t_{2}\right)+\tilde{C}_{4}\left(\xi_{1}+\frac{t_{3}-t_{2}}{2}, t_{3}\right) .
\end{aligned}
$$


Using the above notations, we get from (14) the following relation:

$$
\begin{gathered}
h\left(\xi_{1}, \frac{t_{3}+t_{2}}{2}\right)=\left(W_{1} h\right)\left(\xi_{1}-\frac{t_{3}-t_{2}}{2}, t_{2}\right)+\left(W_{2} h\right)\left(\xi_{1}-\frac{t_{3}-t_{2}}{2}, t_{3}\right) \\
+\left(W_{3} h\right)\left(\xi_{1}+\frac{t_{3}-t_{2}}{2}, t_{2}\right)+\left(W_{4} h\right)\left(\xi_{1}+\frac{t_{3}-t_{2}}{2}, t_{3}\right) .
\end{gathered}
$$

It is final construction.

Remark 11. It seems very strange but the equations (9) and (13) are distinct. One of these equations has a parameter $\xi_{1}$ and the other one has a parameter $\xi_{2}$.

\subsection{A ray in a plane}

This case was first considered in authors' paper (Sh.H. Kutaiba, V.B. Vasilyev, Asymptotic behavior of a solution of a certain boundary value problem, Ital. J. Pure Appl. Math., in press).

\section{Conclusion}

We have considered here some possible cuts in a space. There are lot of other possible cuts which can be constructed from different complicated cones. We will describe these singularities in forthcoming papers.

These studies will help us to describe sufficient conditions under which a general elliptic pseudo-differential equation has Fredholm property and will help us to enlarge the class of manifolds with a singular boundary including considered singular points.

Acknowledgements. This work was supported by the State contract of the Russian Ministry of Education and Science (Contract No FZWG-20200029).

\section{References}

[1] S. Bochner, W.T. Martin, Several Complex Variables, Princeton University Press, Princeton (1948). 
[2] V.S. Vladimirov, Methods of the Theory of Functions of Many Complex Variables, Dover Publications, Mineola, NY (1966).

[3] G. Eskin, Boundary Value Problems for Elliptic Pseudodifferential Equations, Amer. Math. Soc., Providence, RI (1981).

[4] F.D. Gakhov, Boundary Value Problems, Dover Publications, Mineola, NY (1981).

[5] L. Hörmander, The Analysis of Linear Partial Differential Operators III,IV, Springer, Berlin-Heidelberg (1985).

[6] S.G. Milkhin, S. Prößdorf, Singular Integral Operators, Akademie-Verlag, Berlin (1986).

[7] N.I. Muskhelishvili, Singular Integral Equations, North Holland, Amster$\operatorname{dam}(1976)$.

[8] V.B. Vasil'ev, Wave Factorization of Elliptic Symbols: Theory and Applications. Introduction to the Theory of Boundary Value Problems in NonSmooth Domains, Kluwer Academic Publishers, Dordrecht-Boston-London (2000).

[9] S.A. Nazarov, B.A. Plamenevsky, Elliptic Problems in Domains with Piecewise Smooth Boundaries, Walter de Gruyter, Berlin-New York (1994).

[10] L. Rodino, B.-W. Schulze, M.W. Wong (Eds.), Pseudo-Differential Operators: Partial Differential Equations and Time-Frequency Analysis, Fields Institute Communications, Amer. Math. Soc., Providence (2007).

[11] L. Rodino, M.W. Wong (Eds.), Pseudo-Differential Operators: Quantization and Signals, Lect. Notes Math. 1949 (2008).

[12] Ju.V. Egorov, B.-W. Schulze, Pseudo-Differential Operators, Singularities, Applications, Birkhäuser-Verlag, Basel (1997).

[13] B.-W. Schulze, Boundary Value Problems and Singular Pseudo-Differential Operators, Wiley, Chichester (1998).

[14] V.B. Vasilyev, Pseudo-differential equations and conical potentials: 2dimensional case, Opusc. Math., 39 (2019), 109-124.

[15] V.B. Vasilyev, Pseudo-differential equations, wave factorization, and related problems, Math. Methods Appl. Sci., 41 (2018), 9252-9263. 
[16] V.B. Vasilyev, On certain 3D limit boundary value problem. Lobachevskii J. Math., 41 (2020), 913-921. 
